\title{
An Embedding Theorem for Pseudoconvex Domains in Banach Spaces*
}

\author{
Aaron Zerhusen \\ Department of Mathematics \\ Purdue University \\ West Lafayette, IN 47907-2067, USA \\ azerhus@math.purdue.edu
}

\begin{abstract}
We show that a pseudoconvex open subset of a Banach space with an unconditional basis is biholomorphic to a closed direct submanifold of a Banach space with an unconditional basis.
\end{abstract}

\section{Introduction}

A fundamental result in complex geometry, shown by Bishop, Narasimhan and Remmert, is that an $n$-dimensional Stein manifold is biholomorphic to a closed submanifold of $\mathbb{C}^{2 n+1}[\mathrm{~B}, \mathrm{~N}, \mathrm{Re}]$. One reason that this is of such importance is in the study of holomorphic functions on manifolds. Cartan's Theorem B implies that a holomorphic function on a closed submanifold $M \subset \mathbb{C}^{k}$ extends to a function holomorphic on all of $\mathbb{C}^{k}$. Therefore, once a given Stein manifold is embedded in

* This research supported in part by NSF grant DMS-0203072 and a Bilsland Dissertation Fellowship from the Graduate School of Purdue University. This paper is the result of my thesis research under the direction of László Lempert, and I would like to thank him for his patient and wise guidance. I would also like to thank Andreas Defant and David Pérez-García for their suggestions regarding tensor products and directing me to the paper [GR], and Steve Bell for his suggestions. 
some $\mathbb{C}^{k}$, its holomorphic functions can be studied through holomorphic functions on $\mathbb{C}^{k}$. In [L2] Lempert showed a result similar to the Bishop-Narasimham-Remmert embedding theorem for Banach spaces, namely, if $\Omega$ is a pseudoconvex domain in a Banach space $X$, and $X$ has an unconditional basis, then $\Omega$ is biholomorphic to a closed direct submanifold of a separable Banach space $Y$. However, his proof does not guarantee that $Y$ would have an unconditional basis or Hilbert space structure if $X$ does. This is an important issue, since many complex analytic results, such as approximation theorems, rely upon the existence of an unconditional basis. In particular, in [L3] and $[\mathrm{P}]$ it is shown that if $M$ is a closed direct submanifold in $Y$, a Banach space with unconditional basis, then holomorphic functions on $M$ extend to holomorphic functions on $Y$. In this paper we resolve the above issue by improving upon Lempert's embedding theorem. Our main theorem is

Theorem 1.1. Let $X$ be a Banach space with an unconditional basis, and $\Omega \subset X$ pseudoconvex and open. Then $\Omega$ is biholomorphic to a closed direct submanifold of a Banach space $Y$, which has an unconditional basis. Further, if $1<p<\infty$ and $X$ is a separable $L^{p}$ space, then $Y$ can also be taken to be a separable $L^{p}$ space.

Just as in [L2], this is an immediate consequence of a domination theorem.

Theorem 1.2. Let $X$ be a Banach space with an unconditional basis, $\Omega \subset X$ pseudoconvex and open, and $u: \Omega \rightarrow \mathbb{R}$ locally bounded. There is a Banach space $V$ with unconditional basis and a holomorphic $f: \Omega \rightarrow V$ such that $u(x) \leq\|f(x)\|$ for $x \in \Omega$. If $1<p<\infty$ and $X$ is a separable $L^{p}$ space, then $V$ may also be taken to be a separable $L^{p}$ space.

Proof of Theorem 1.1 from Theorem 1.2. Let $d: \Omega \rightarrow \mathbb{R}, d(x)=$ $\operatorname{dist}(x, \partial \Omega)$, and let $f \in \mathcal{O}(\Omega, V)$ such that $\|f(x)\| \geq \frac{1}{d(x)}$. Then the graph of $f$ is a closed direct submanifold of $X \times V$ which is biholomorphic to $\Omega$. This proves the theorem, since if $X$ and $V$ have unconditional bases so does $X \times V$, and if $X$ and $V$ are $L^{p}$ spaces so is $X \times V$. 


\section{Definitions}

We will require some notions from the theory of Banach spaces. A Schauder basis for a Banach space $X$ (for this paper, we always mean over $\mathbb{C}$ ) is a countable sequence of vectors $e_{1}, e_{2}, \ldots \in X$ such that for every $x \in X$ there is a unique sequence of complex numbers $\lambda_{1}, \lambda_{2}, \ldots \in \mathbb{C}$ such that

$$
x=\sum \lambda_{n} e_{n}
$$

where the sum converges in norm. Having a Schauder basis implies that the space is separable. If the sequence converges in norm after arbitrary rearrangements, it is called an unconditional basis, or equivalently, if $\sum_{1}^{\infty} \lambda_{n} \theta_{n} e_{n}$ converges whenever $\sum_{1}^{\infty} \lambda_{n} e_{n}$ does, for all choices of $\theta_{n}= \pm 1$. Following [LT], for $x=\sum_{1}^{\infty} \lambda_{n} e_{n}$ and every $\theta=$ $\left\{\theta_{n}\right\}_{n=1}^{\infty}, \theta_{n}= \pm 1$ define a linear operator $M_{\theta} x=\sum_{1}^{\infty} \lambda_{n} \theta_{n} e_{n}$. The principle of uniform boundedness guarantees that $c=\sup _{\theta}\left\|M_{\theta}\right\|<\infty$, and we call $c$ the unconditional constant of the basis. We call a basis with unconditional constant 1 a 1-unconditional basis. Upon renorming with an equivalent norm, any space with an unconditional basis has a 1-unconditional basis [LT, p. 18]. The usual basis of $l^{p}$ for $1 \leq p<\infty$ and the Haar basis of $L^{p}[0,1]$ for $1<p<\infty$ are examples of unconditional bases (although the Haar basis is not 1-unconditional). $L^{1}[0,1]$, however does not have an unconditional basis. Since any separable $L^{p}$ space is isometrically isomorphic to $l_{n}^{p}, l^{p}, L^{p}[0,1]$, or a direct sum of these, for $1<p<\infty$ any separable $L^{p}$ space has an unconditional basis. With a choice of basis $\left\{e_{n}\right\}$ for a Banach space $X$ we associate a set of projections. Let $\pi_{N}: X \rightarrow X$ be the projection $\pi_{N} \sum_{n=1}^{\infty} \lambda_{n} e_{n}=\sum_{n=1}^{N} \lambda_{n} e_{n}$ and $\rho_{N}=\mathrm{id}-\pi_{N}$. If $\left\|\pi_{N}\right\|=1$ for all $N$, we say that $\left\{e_{n}\right\}$ is monotone. It is immediate that every 1 -unconditional basis is monotone, and it is also true that the Haar basis is a monotone basis for $L^{p}(0,1)$ for all $1 \leq p<\infty$ [LT, p. 3]. Therefore, for all $1<p<\infty$ any separable $L^{p}$ space has a monotone unconditional basis.

We will also require certain notions from the theory of complex analysis in Banach spaces. For more on this subject, see [D or $\mathrm{M}]$. For an open set $\Omega \subset X$ to be pseudoconvex means that $\Omega \cap Y$ is pseudoconvex in the usual sense for all finite dimensional subspaces $Y \subset X$. A Hausdorff space $N$ is a complex manifold modeled on a Banach space $Z$ if $N$ has an open cover $\left\{U_{\alpha}\right\}$, for each $\alpha$ we specify a homeomorphism $\phi_{\alpha}$ from $U_{\alpha}$ to an open subset of $Z$, and $\phi_{\alpha} \phi_{\beta}^{-1}$ are 
holomorphic where defined. For $M \subset N$ to be a complex submanifold means that as a pair $(M, N)$ is locally biholomorphic to $(X, Z), X$ a Banach subspace of $Z$. If $X$ is a complemented subspace of $Z$ we say that $M$ is a direct submanifold of $N$.

\section{The Proof of Theorem 1.2}

Let $\mathcal{B}$ denote the covering of $\Omega \subset X$

$$
\mathcal{B}=\mathcal{B}_{\Omega}=\{\text { balls } B: \bar{B} \subset \Omega, 2 \operatorname{diam} B<\operatorname{diam} \Omega\}
$$

Proposition 3.1. Suppose either that (a) $X$ is a Banach space with a 1-unconditional basis, or (b) $1<p<\infty$ and $X$ is a separable $L^{p}$ space. Given $\Omega \subset X$ pseudoconvex open, and $u: \Omega \rightarrow \mathbb{R}$, suppose that for all $B \in \mathcal{B}_{\Omega}$ there are a Banach space $V_{B}$ with 1-unconditional basis in case (a), separable $L^{p}$ space in case (b), and $f_{B} \in \mathcal{O}\left(B ; V_{B}\right)$ such that $u(x) \leq\left\|f_{B}(x)\right\|_{V_{B}}$ for all $x \in B$. Then there are a Banach space $V$ and $f \in \mathcal{O}(\Omega, V)$ such that in case (a) $V$ has 1-unconditional basis, in case (b) $V$ is a separable $L^{p}$ space, and $u(x) \leq\|f(x)\|_{V}$ for all $x \in \Omega$.

Proof of Theorem 1.2 from Proposition 3.1. This proof is the same as in [L2]. Let $u: \Omega \rightarrow \mathbb{R}$ be locally bounded and assume that on $\Omega$ holomorphic domination of $u$ with values in a space with 1-unconditional basis (respectively, a separable $L^{p}$ space) is not possible. Then, by Proposition 3.1, there exists a ball $B_{1} \subset \Omega$ such that diam $B_{1}<1 / 2$ $\operatorname{diam} \Omega$, on which holomorphic domination is not possible. By again applying Proposition 3.1, but with $B_{1}$ instead of $\Omega$, there exists a ball $B_{2}, \bar{B}_{2} \subset B_{1}$ with diam $B_{2}<\operatorname{diam} B_{1} / 2$, on which $u$ cannot be dominated by a holomorphic function. Continuing in this way we get a descending sequence of balls on which holomorphic domination is impossible. Let $x=\bigcap_{1}^{\infty} B_{j}$. Thus holomorphic domination is not possible on any neighborhood of $x$, contradicting the local boundedness of $u$.

\section{Exhaustion techniques}

The rest of this paper is devoted to proving Proposition 3.1. Let $X$ be as there; $e_{1}, e_{2}, \ldots$ denote, in case (a), a 1-unconditional basis; in 
case (b), a monotone unconditional basis. Recall the projections $\pi_{N}$ and $\rho_{N}$ from Section 2 .

To prove Theorem 1.2 or Proposition 3.1 in the finite dimensional setting, a reasonable technique would be to exhaust $\Omega$ by compact sets. In the setting of an infinite dimensional Banach space, this is not possible as any compact set has empty interior. Lempert proposes instead exhausting by ball bundles of a certain type. The definitions and theorems in this section are from [L2]. In order to exhaust $\Omega$, we consider a special type of ball bundle. Let

$$
d(x)=\min \{1, \operatorname{dist}(x, X \backslash \Omega)\},
$$

and $0<\alpha<1$. For any positive integer $N$, define

$$
\begin{gathered}
D_{N}\langle\alpha\rangle=\left\{t \in \pi_{N} X:\|t\|<\alpha N, 1<\alpha N d(t)\right\} \\
\Omega_{N}\langle\alpha\rangle=\left\{x \in X: \pi_{N} x \in D_{N}\langle\alpha\rangle,\left\|\rho_{N} x\right\|<\alpha d\left(\pi_{N} x\right)\right\} .
\end{gathered}
$$

Theorem 4.1. a. Each $\Omega_{N}\langle\alpha\rangle$ is pseudoconvex.

b. $\overline{\Omega_{n}\langle\gamma\rangle} \subset \Omega_{N}\langle\beta\rangle$ if $n \leq N, \gamma \leq \beta / 4$.

c. For fixed $\gamma$ each $x \in \Omega$ has a neighborhood that is contained in all but finitely many $\Omega_{N}\langle\gamma\rangle$.

This is proven in [L2, Proposition 3.1]. Note that in the proof of (b) only the monotonicity of the basis is used.

Just as in many situations in the theory of finitely many variables, we need a result regarding holomorphic approximation on certain sets.

Lemma 4.2. Assume $X$ has an unconditional basis with basis constant $c$. If $\gamma<2^{-7} \alpha / c$ and $V$ is a Banach space, then any $\psi \in$ $\mathcal{O}\left(\Omega_{N}\langle\alpha\rangle ; V\right)$ can be approximated by $\phi \in \mathcal{O}(\Omega ; V)$, uniformly on $\Omega_{N}\langle\gamma\rangle$.

We first consider the simpler case of balls about the origin. Let $B(\mu)=\{x \in X:\|x\|<\mu\}$.

Lemma 4.3. Assume $X$ has an unconditional basis with basis constant $c$. Then for any Banach space $\left(W,\|\|_{W}\right), \epsilon>0$, and $g \in$ $\mathcal{O}(B(1) ; W)$ there is an $h \in \mathcal{O}(X ; W)$ that satisfies $\|g-h\|<\epsilon$ on $B(1 /(2 c))$. 
Proof. It was shown by Lempert in [L1] and by Josefson in $[\mathrm{J}]$ that every space with an unconditional basis allows such approximation on a ball when $c=1$. Let $\|x\|^{\prime}=\sup _{\theta}\left\|M_{\theta} x\right\|$. This is a norm equivalent to \|\| under which $e_{1}, e_{2}, \ldots$ is a 1 -unconditional basis. Let $B^{\prime}(\mu)=\left\{x \in X:\|x\|^{\prime}<\mu\right\}$. For all $x \in X$ we have the inequality

$$
\|x\| \leq\|x\|^{\prime} \leq c\|x\|,
$$

which gives that $B(1 /(2 c)) \subset B^{\prime}(1 / 2)$ and $B^{\prime}(1) \subset B(1)$. The lemma is therefore an immediate consequence of the Josefson-Lempert approximation results.

Now the proof of Lemma 4.2 is a special case of [L2, Theorem 3.3] with $\mu=1 /(2 c)$.

\section{Tensor products and sums of Ba- nach spaces}

In the proof of Proposition 3.1, we will use tensor products of Banach spaces. For the general theory of tensor products see [DF or Ry]. If $X$ and $Y$ are Banach spaces, as usual $X \otimes Y$ will denote the tensor product of the underlying complex vector spaces. We will use $X \bar{\otimes} Y$ to denote the completion of $X \otimes Y$ with respect to a certain norm which preserves key features of $X$ and $Y$ in the following sense. If $X=L^{p}(\mu)$ and $Y=L^{p}(\nu)$, we define the norm of $\sum_{1}^{n} x_{j} \otimes y_{j} \in X \otimes Y$ by

$$
\left\|\sum_{1}^{n} x_{j} \otimes y_{j}\right\|=\left(\int\left|\sum_{1}^{n} x_{j}(s) y_{j}(t)\right|^{p} d \mu(s) d \nu(t)\right)^{1 / p} .
$$

In particular, Fubini's theorem gives

$$
\|x \otimes y\|=\|x\|\|y\| .
$$

Under this norm $X \bar{\otimes} Y$ is isometrically isomorphic to $L^{p}(\mu \times \nu)[\mathrm{DF}$ p.79].

If $X$ and $Y$ are spaces with 1-unconditional bases $\left(e_{n}\right)$ and $\left(f_{n}\right)$ respectively, Grecu and Ryan define a norm on $X \otimes Y$ with the property that $\left(e_{n} \otimes f_{m}\right)$ is a 1-unconditional basis for the completion $X \bar{\otimes} Y[\mathrm{GR}]$. How they define this norm and other properties it has are not of immediate importance to us, just that it preserves unconditional structure and also satisfies (5.2). 
We now look at certain facts about sums of Banach spaces. If $\left(W_{m}\right)$ is a sequence of Banach spaces, each with 1-unconditional basis, or each an $L^{p}$ space, let

$$
W=\bigoplus_{m=0}^{\infty} W_{m}
$$

where the bar represents the completion with respect to the $l^{p}$ norm if $W_{m}$ are $L^{p}$ spaces or the $l^{1}$ norm if they are spaces with 1-unconditional basis. Concretely,

$$
W=\left\{w=\left(w_{m}\right): w_{m} \in W_{m},\|w\|=\left(\sum_{m=0}^{\infty}\left\|w_{m}\right\|^{p}\right)^{1 / p}<\infty .\right\}
$$

Clearly, $W$ is a separable Banach space.

Lemma 5.1. 1. If $W_{m}$ all have 1-unconditional bases, so does $W$.

2. If $W_{m}$ are all $L^{p}$ spaces, so is $W$.

Proof. Let $\left(e_{m n}\right)_{n=1}^{\infty}$ be a 1-unconditional basis for $W_{m}, \theta=\left(\theta_{m n}\right), \theta_{m n}=$ \pm 1 . If $x \in W, x=\sum \lambda_{m n} e_{m n}$.

$$
\begin{aligned}
\left\|\sum \theta_{m n} \lambda_{m n} e_{m n}\right\| & =\left(\sum_{m=0}^{\infty}\left\|\sum_{n=0}^{\infty} \theta_{m n} \lambda_{m n} e_{m n}\right\|\right) \\
& \leq\left(\sum_{m=0}^{\infty}\left\|\sum_{n=0}^{\infty} \lambda_{m n} e_{m n}\right\|\right) \\
& \leq\|x\|
\end{aligned}
$$

So $\left(e_{m n}\right)$ is a 1-unconditional basis for $W$. If $W_{m}$ are $L^{p}$ spaces, $W$ is isometrically isomorphic to the corresponding $L^{p}$ space on the disjoint union of the appropriate measure spaces.

\section{The proof of Proposition 3.1}

Fix $N \in \mathbb{N}$, and let $\pi=\pi_{N+1}$ and $\rho=\rho_{N+1}$. If $A \subset \pi X$ and $r: A \rightarrow \mathbb{R}$ is continuous, then ball bundles of the form

$$
A(r)=\{x \in X: \pi x \in A,\|\rho x\|<r(\pi x)\}
$$




$$
A[r]=\{x \in X: \pi x \in A,\|\rho x\| \leq r(\pi x)\}
$$

are called sets of type B. Note that the sets $\Omega_{N}\langle\alpha\rangle$ are specific examples of sets of type B.

Lemma 6.1. Assume either that (a) $X$ is a Banach space with 1unconditional basis or (b) $1<p<\infty$ and $X$ is a separable $L^{p}$ space. Let $\Omega \subset X$ be open and $A_{2} \subset \subset A_{3} \subset \subset A_{4}$ be relatively open subsets of $\Omega \cap \pi X, A_{1} \subset A_{2}$ compact and plurisubharmonically convex in $A_{4}$. Let $r_{i}: A_{4} \rightarrow(0, \infty)$ be continuous, $1 \leq i \leq 4$, and $r_{i}<r_{i+1}$, with $-\log r_{1}$ plurisubharmonic. Assume that any Banach space valued holomorphic function on $A_{4}\left(r_{4}\right)$ can be uniformly approximated on $A_{3}\left(r_{3}\right)$ by functions holomorphic on $\Omega$. Then there exist a Banach space $W$ and a function $\psi \in \mathcal{O}(\Omega, W)$ such that in case (a) W has a 1-unconditional basis, in case (b) $W$ is a separable $L^{p}$ space, and

1. $\|\psi(x)\| \leq 1 / 2$ if $x \in A_{1}\left[r_{1}\right]$

2. $\|\psi(x)\| \geq 2$ if $x \in A_{3}\left(r_{3}\right) \backslash A_{2}\left(r_{2}\right)$.

Proof. Define Hartogs sets in $\pi X \times \mathbb{C} \approx \mathbb{C}^{N+2}$ :

$$
\begin{gathered}
H_{1}=\left\{(s, \lambda) \in A_{1} \times \mathbb{C}:|\lambda| \leq r_{1}(s)\right\} \\
H_{i}=\left\{(s, \lambda) \in A_{i} \times \mathbb{C}:|\lambda|<r_{i}(s)\right\}, 2 \leq i \leq 4 .
\end{gathered}
$$

The set $H_{1}$ is plurisubharmonically convex, hence holomorphically convex, in $H_{4}[\mathrm{H}]$. Since $\bar{H}_{3} \backslash H_{2}$ is compact, there exist finitely many holomorphic functions $\phi_{j} \in \mathcal{O}\left(H_{4}\right), j=1, \ldots, J$, such that if $z \in H_{1}$ we have $\left|\phi_{j}(z)\right|<1 / 4$ for all $j$ and if $z \in H_{3} \backslash H_{2}$ there is a $j$ such that $\left|\phi_{j}(z)\right|>4$. There exists a positive $\eta<1$ such that with

$$
\begin{gathered}
H_{1}^{\prime}=\left\{(s, \lambda) \in A_{1} \times \mathbb{C}:|\lambda| \leq \eta^{-1} r_{1}(s)\right\} \\
H_{2}^{\prime}=\left\{(s, \lambda) \in A_{2} \times \mathbb{C}:|\lambda|<\eta r_{i}(s)\right\},
\end{gathered}
$$

$\left|\phi_{j}(z)\right|<1 / 2$ for all $j$ if $z \in H_{1}^{\prime}$ and $\left|\phi_{j}(z)\right|>2$ for some $j$ if $z \in$ $H_{3} \backslash H_{2}^{\prime}$. By replacing $\phi_{j}$ with $\phi_{j}^{n}$ for a suitably large $n$ we can assume that $\left|\phi_{j}(z)\right|<(1-\eta) /(4 J)$ for all $j$ if $z \in H_{1}^{\prime}$ and $\left|\phi_{j}(z)\right|>4 /(1-\eta)$ for some $j$ for each $z \in H_{3} \backslash H_{2}^{\prime}$. Let $W^{\prime}=\bar{\oplus}_{n=0}^{\infty} X^{\bar{\otimes} n}$, taking $X^{\bar{\otimes} 0}$ to be $\mathbb{C}$. As shown before, $W^{\prime}$ has a 1-unconditional basis if $X$ does and $W^{\prime}$ is a separable $L^{p}$ space if $X$ is. Since $\phi_{j}$ is holomorphic, $\phi_{j}(s, \lambda)=$ $\sum_{n=0}^{\infty} a_{n j}(s) \lambda^{n}$, where $a_{n j}$ depends holomorphically on $s \in A_{4}$ and 
locally on $H_{4}$ convergence is absolute and uniform. Define $\psi_{j}$ from $A_{4}\left(r_{4}\right)$ to $W^{\prime}$ as

$$
\psi_{j}(x)=\left(a_{n j}(\pi x)(\rho x)^{\otimes n}\right)_{n=0}^{\infty} .
$$

By the absolute and uniform convergence of the power series of $\phi_{j}, \psi_{j}$ is holomorphic.

For any $x \in A_{4}\left(r_{4}\right)$ and any $j=1, \ldots, J$, by definition of the norm of $W^{\prime}$ (and since the $l^{1}$ norm dominates the $l^{p}$ norm for $p \geq 1$ )

$$
\left\|\psi_{j}(x)\right\| \leq \sum_{n=0}^{\infty}\left|a_{n j}(\pi x)\right|\|\rho x\|^{n} .
$$

If $x \in A_{1}\left[r_{1}\right]$,

$$
\left\|\psi_{j}(x)\right\| \leq \sum_{n=0}^{\infty}\left|a_{n j}(\pi x)\right|\left(r_{1}(\pi x)\right)^{n} .
$$

Fixing $s \in A_{1}$ and using Cauchy estimates for $\psi_{j}$ on $|\lambda|=\eta^{-1} r_{1}(s)$ we see that $\left|a_{n j}(s)\right| \leq(1-\eta)\left(\eta / r_{1}(s)\right)^{n} /(4 J)$, so

$$
\left\|\psi_{j}(x)\right\| \leq(1-\eta) /(4 J) \sum_{n=0}^{\infty} \eta^{n}=1 /(4 J) .
$$

Next let $x \in A_{3}\left(r_{3}\right) \backslash A_{2}\left(r_{2}\right)$ so that $(\pi x,\|\rho x\|) \in H_{3} \backslash H_{2}$. In case (a), $W^{\prime}$ is an $l^{1}$ sum and it is immediate that $\left\|\psi_{j}(x)\right\| \geq\left|\phi_{j}(\pi x,\|\rho x\|)\right|$, hence $\left\|\psi_{j}(x)\right\|>4$ for some $j$. In case (b), i.e. $X$ is an $L^{p}$ space, choose $j$ so that $\left|\phi_{j}(\pi x, \eta\|\rho x\|)\right|>4 /(1-\eta)$. By Hölder's inequality:

$$
\begin{aligned}
\left\|\psi_{j}(x)\right\| & =\left(\sum_{n=0}^{\infty}\left(\left|a_{n j}(\pi x)\right|\|\rho x\|^{n}\right)^{p}\right)^{1 / p} \\
& \geq\left(\sum_{n=0}^{\infty}\left|a_{n j}(\pi x)\right|\|\eta \rho x\|^{n}\right)\left(\sum_{n=0}^{\infty} \eta^{n q}\right)^{-1 / q} \\
& \geq\left|\phi_{j}(\pi x, \eta\|\rho x\|)\right|\left(\sum_{n=0}^{\infty} \eta^{n}\right)^{-1} \\
& >4 .
\end{aligned}
$$

Define $W=\oplus_{j=1}^{J} W^{\prime}$ and $\tilde{\psi}(x)=\left(\psi_{1}(x), \ldots \psi_{J}(x)\right)$. Let $\psi \in \mathcal{O}(\Omega, W)$ approximating $\tilde{\psi}$ within $1 / 4$ on $A_{3}\left(r_{3}\right)$. Then $\|\psi(x)\| \leq 1 / 2$ if $x \in$ $A_{1}\left[r_{1}\right]$, and $\|\psi(x)\| \geq 2$ if $x \in A_{3}\left(r_{3}\right) \backslash A_{2}\left(r_{2}\right)$. 
In the next lemma the geometric assumptions are the same as in the previous one.

Lemma 6.2. Assume either that (a) $X$ and $Y$ are Banach spaces with 1-unconditional basis or (b) $1<p<\infty$ and $X$ and $Y$ are separable $L^{p}$ spaces. Let $\Omega \subset X$ is open and $A_{2} \subset \subset A_{3} \subset \subset A_{4}$ be relatively open subsets of $\Omega \cap \pi X, A_{1} \subset A_{2}$ compact and plurisubharmonically convex in $A_{4}$. Let $r_{i}: A_{4} \rightarrow(0, \infty)$ be continuous, $1 \leq i \leq 4$ and $r_{i}<r_{i+1}$ with $-\log r_{1}$ plurisubharmonic. Assume that any Banach space valued holomorphic function on $A_{4}\left(r_{4}\right)$ can be uniformly approximated on $A_{3}\left(r_{3}\right)$ by functions holomorphic on $\Omega$. Let $\epsilon>0$. Then given a function $g \in \mathcal{O}(X, Y)$, there exist a Banach space $Z$ and a function $h \in \mathcal{O}(\Omega, Z)$ such that in case (a) $Z$ has a 1-unconditional basis, in case (b) $Z$ is a separable $L^{p}$ space, and

1. $\|h(x)\| \leq \epsilon$ if $x \in A_{1}\left[r_{1}\right]$

2. $\|h(x)\| \geq\|g(x)\|$ if $x \in A_{3}\left(r_{3}\right) \backslash A_{2}\left(r_{2}\right)$.

Proof. Without loss of generality, assume $\|g(x)\| \geq \epsilon$ for all $x \in$ $A_{4}\left(r_{4}\right)$. By Lemma 6.1, there are a Banach space $W$ with 1-unconditional basis (or an $L^{p}$ space, as appropriate) and a function $\psi \in \mathcal{O}(\Omega, W)$ with $\|\psi(x)\| \leq 1 / 2$ for $x \in A_{1}\left[r_{1}\right]$ and $\|\psi(x)\| \geq 2$ if $x \in A_{3}\left(r_{3}\right) \backslash$ $A_{2}\left(r_{2}\right)$. We can expand $g$ in a series

$$
g(x)=g(\pi x+\rho x)=\sum_{m=0}^{\infty} g_{m}(\pi x+\rho x)
$$

where the $g_{m}$ 's are $m$-homogeneous in $\rho x$, and convergence is uniform on compact subsets of $X$. There exist $\delta>0$ and $M \geq 0$ such that $\pi x \in A_{3}$ and $\|\rho x\| \leq \delta$ imply $\|g(x)\| \leq M$, hence

$$
\left\|g_{m}(x)\right\|=\left\|\frac{1}{2 \pi i} \int_{|\zeta|=\delta} \frac{g(\pi x+\zeta \rho x)}{\zeta^{m+1}} d \zeta\right\| \leq \delta^{-m} M .
$$

With $\alpha=\alpha_{1}+\alpha_{2}$ and $\beta$ (sufficiently large) integers to be specified later, define functions from $A_{4}\left[r_{4}\right]$ to $Z_{m}=Y \bar{\otimes} W^{\bar{\otimes}(\alpha m+\beta)}$ by

$$
h_{m}(x)=g_{m}(x) \otimes \psi(x)^{\otimes(\alpha m+\beta)} .
$$

Note that each $h_{m}$ is holomorphic. We will show that $\tilde{h}=\left(h_{m}\right)_{m=0}^{\infty}$ defines a holomorphic map $A_{4}\left(r_{4}\right) \rightarrow Z=\bar{\bigoplus}_{m=0}^{\infty} Z_{m}$. Indeed, if $K \subset$ $A_{4}\left(r_{4}\right)$ is compact and $x \in K$,

$$
\|\tilde{h}(x)\|^{p} \leq \sum_{m=0}^{\infty}\left(\left\|g_{m}(x)\right\|\left(\|\psi(x)\|^{\alpha m+\beta}\right)^{p}\right.
$$




$$
\leq M \sum_{m=0}^{\infty}\left\|g_{m}\left(\pi x+M^{\alpha} \rho x\right)\right\|^{p},
$$

(here and below, we use $p=1$ in case (a)). Now $\tilde{K}=\pi K+M^{\alpha} \rho K$ being compact and $g$ entire,

$$
M \sum_{m=0}^{\infty}\left\|g_{m}\left(\pi x+M^{\alpha} \rho x\right)\right\|^{p}
$$

converges uniformly on $K$. Thus $\tilde{h}$, being a limit of holomorphic functions which is uniform on compact sets, is holomorphic on $A_{4}\left(r_{4}\right)$.

To estimate $\tilde{h}(x)$ for $x \in A_{3}\left(r_{3}\right) \backslash A_{2}\left(r_{2}\right)$ we use Hölder's inequality:

$$
\begin{aligned}
\|\tilde{h}(x)\| & =\left(\sum_{m=0}^{\infty}\left(\left\|g_{m}(x)\right\|\|\psi(x)\|^{\alpha m+\beta}\right)^{p}\right)^{1 / p} \\
& \geq\left(\sum_{m=0}^{\infty}\left(\left\|g_{m}(x)\right\| 2^{\alpha m+\beta}\right)^{p}\right)^{1 / p} . \\
& \geq\left(\sum_{m=0}^{\infty}\left\|g_{m}(x)\right\|\right)\left(\sum_{m=0}^{\infty}(1 / 2)^{(\alpha m+\beta) q}\right)^{-1 / q}
\end{aligned}
$$

where $1 / q+1 / p=1$. If $\beta \geq 2$, then

$\|\tilde{h}(x)\| \geq 2 \sum_{m=0}^{\infty}\left\|g_{m}(x)\right\| \geq 2\|g(x)\| \leq\|g(x)\|+\epsilon, \quad x \in A_{3}\left(r_{3}\right) / A_{2}\left(r_{2}\right)$.

Next we estimate $\tilde{h}$ for $x \in A_{1}\left[r_{1}\right]$ :

$$
\begin{aligned}
\|\tilde{h}(x)\| & =\left(\sum_{m=0}^{\infty}\left(\left\|g_{m}(x)\right\|(\|\psi(x)\|)^{\alpha m+\beta}\right)^{p}\right)^{1 / p} \\
& \leq\left(\sum_{m=0}^{\infty}\left\|g_{m}(x) 2^{-(\alpha m+\beta)}\right\|^{p}\right)^{1 / p} \\
& \leq 2^{-\beta}\left(\sum_{m=0}^{\infty}\left(\left\|g_{m}\left(\pi x+2^{-\alpha_{2}} \rho x\right)\right\| 2^{-m \alpha_{1}}\right)^{p}\right)^{1 / p .}
\end{aligned}
$$

Choose $\alpha_{2}$ so that $2^{-\alpha_{2}}\|\rho x\| \leq \delta$ for $x \in A_{1}\left[r_{1}\right]$, then by (6.2), we continue estimating (6.5):

$$
\leq 2^{-\beta}\left(\sum_{m=0}^{\infty}\left(M \delta^{-m} 2^{-m \alpha_{1}}\right)^{p}\right)^{1 / p} .
$$


Now choose the exponent $\alpha_{1}$ so that $2^{-\alpha_{1}} \leq \delta / 2$ and $\beta \geq 2$ so that $2^{-\beta+1} M \leq \epsilon$, giving

$$
\begin{aligned}
\|\tilde{h}(x)\| & \leq 2^{-\beta} M\left(\sum_{m=0}^{\infty}\left(2^{-m-1}\right)^{p}\right)^{1 / p} \\
& \leq \epsilon / 2\left(\sum_{m=0}^{\infty}\left(2^{-m-1}\right)^{p}\right)^{1 / p}<\epsilon / 2 .
\end{aligned}
$$

Therefore $\|\tilde{h}\|<\epsilon / 2$. Let $h \in \mathcal{O}(\Omega, Z)$ such that $h$ approximates $\tilde{h}$ within $\epsilon / 2$ on $A_{3}\left(r_{3}\right)$. Then $\|h(x)\|<\epsilon$ if $x \in A_{1}\left[r_{1}\right]$ and $\|h(x)\|>$ $\|g(x)\|$ if $x \in A_{3}\left(r_{3}\right) \backslash A_{2}\left(r_{2}\right)$.

Proposition 6.3. Let $\epsilon>0$. Assume either that (a) $X$ and $Y$ are Banach spaces with 1-unconditional basis or (b) $X$ and $Y$ are separable $L^{p}$ spaces. Let $2^{4} \beta<\alpha<2^{-9} / c$ where $c$ is the unconditional basis constant of $X$. If $\Omega \subset X$ is pseudoconvex, and $g \in \mathcal{O}(X ; Y)$, then there are a Banach space $Z$ and $h \in \mathcal{O}(\Omega ; Z)$ such that in case (a) $Z$ has 1-unconditional basis, in case (b) $Z$ is a separable $L^{p}$ space, and

1. $\|h(x)\|_{Z} \leq \epsilon$ if $x \in \Omega_{N}\langle\beta\rangle$, and

2. $\|h(x)\|_{Z} \geq\|g(x)\|_{Y}$ if $x \in \Omega_{N+1}\langle\alpha\rangle \backslash \Omega_{N}\langle\alpha\rangle$.

The proof of this theorem depends on showing that sets $A_{i}$ and functions $r_{i}$ can be defined satisfying the conditions of Lemma 6.2 so that $\Omega_{N}\langle\beta\rangle \subset A_{1}\left[r_{1}\right]$ and $\Omega_{N+1}\langle\alpha\rangle \backslash \Omega_{N}\langle\alpha\rangle \subset A_{3}\left(r_{3}\right) \backslash A_{2}\left(r_{2}\right)$. Then we apply Lemma 6.2. These containments are shown in the proof of Proposition 4.2 in [L2], here using $\mu=1 /(2 c)$.

Proof of Proposition [3.1. The first part of the proof is as in [L2]. Let $\alpha<2^{-9} / c$, where $c$ is the unconditional basis constant of $X$. First, for each $N \in \mathbb{N}$ we create a Banach space with a 1-unconditional basis (separable $L^{p}$ space) $Z_{N}$ and a function $g_{N} \in \mathcal{O}\left(X, Z_{N}\right)$ such that $u \leq\left\|g_{N}\right\|_{Z_{N}}$ on $\Omega_{N}\langle\alpha\rangle$. Assume $u>1$ everywhere. Define $A=\overline{\Omega_{N}\langle\alpha\rangle} \cap \pi_{N} X$, a compact set. Recalling the definition of $d(x)$ from (4.1), if $t \in A$, then

$$
\Omega_{N}\langle\alpha\rangle \cap \pi_{N}^{-1} t \subset B(t, \alpha d(t)) .
$$

Therefore, there is a neighborhood $U \ni t, U \subset \pi_{N} X$, such that $\Omega_{N}\langle\alpha\rangle \cap \pi_{N}^{-1} U \subset B(t, 2 \alpha d(t))$. By compactness of $A$, there is a finite set $T \subset A$ such that

$$
\Omega_{N}\langle\alpha\rangle \subset \bigcup_{t \in T} B(t, 2 \alpha d(t))
$$


Now we show that $B_{t}=B(t, 4 c \alpha d(t)) \in \mathcal{B}$. Indeed,

$$
2 \operatorname{diam} B_{t}=16 \operatorname{cod}(t)<2 d(t) \leq \operatorname{diam} \Omega
$$

So by the hypothesis of Proposition 3.1 there are a Banach space $V_{t}$ with 1-unconditional basis (separable $L^{p}$ space) and a function $f_{t} \in$ $\mathcal{O}\left(B_{t}, V_{t}\right)$ such that $u \leq\left\|f_{t}\right\|_{V_{t}}$ on $B_{t}$. By Lemma 4.3 concerning approximation on balls, there are $f_{t}^{\prime} \in \mathcal{O}\left(X, V_{t}\right)$ such that $\| 2 f_{t}-$ $f_{t}^{\prime} \|_{V_{t}}<1$ on $B(t, 2 \alpha d(t))$; so $u \leq\left\|f_{t}^{\prime}\right\|_{V_{t}}$ on $B(t, 2 \alpha d(t))$. Define $Z_{N}=\bigoplus_{t \in T} V_{t}$ with the $l^{1}$ norm in case (a) or $l^{p}$ norm in case (b), and $g_{N}=\bigoplus_{t \in T} f_{t}^{\prime} \in \mathcal{O}\left(X, Z_{N}\right)$. Then since the sets $B(t, 2 \alpha d(t))$ cover $\Omega_{N}\langle\alpha\rangle$, given $x \in \Omega_{N}\langle\alpha\rangle$, there is a $t$ such that $\left\|f_{t}(x)\right\| \geq u(x)$, so $u(x) \leq\left\|g_{N}(x)\right\|_{Z_{N}}$ for all $x \in \Omega_{N}\langle\alpha\rangle$.

Fix $\beta<2^{-4} \alpha$. By using Proposition 6.3, we have $Y_{N}$ a Banach space with 1-unconditional basis (separable $L^{p}$ space) and $h_{N} \in$ $\mathcal{O}\left(\Omega, Y_{N}\right)$ satisfying $\left\|h_{N}\right\|_{Y_{N}} \leq 2^{-N}$ on $\Omega_{N}\langle\beta\rangle$ and $\left\|h_{N}\right\|_{Y_{N}} \geq\left\|g_{N}\right\|_{Z_{N}} \geq$ $u$ on $\Omega_{N+1}\langle\alpha\rangle \backslash \Omega_{N}\langle\alpha\rangle$. Let $V=\bar{\bigoplus}_{N=0}^{\infty} Y_{N}$. Since any $x$ in $\Omega$ is in $\Omega_{N}\langle\beta\rangle$ for all but finitely many $N, \sum_{N}\left\|h_{N}\right\|$ converges locally uniformly, implying that $f=\bigoplus_{N} h_{N}$ is in $\mathcal{O}(\Omega, V)$. For any $x \in \Omega$, there is an $N$ so that $x \in \Omega_{N+1}\langle\alpha\rangle \backslash \Omega_{N}\langle\alpha\rangle$, then $\|f(x)\|_{V} \geq\left\|h_{N}(x)\right\|_{Y_{N}} \| \geq$ $u(x)$, and $\|f\|_{V} \geq u$ on $\Omega$.

\section{References}

[B] E. Bishop, Mappings of partially analytic spaces, Amer. J. Math. 83 (1961), 209-242.

[DF] A. Defant, K. Floret, Tensor Norms and Operator Ideals, NorthHolland Mathematics Studies. 176. Amsterdam: North-Holland. xi, 566 p. (1993).

[D] S. Dineen, Complex Analysis on Infinite Dimensional Spaces, Springer Monographs in Mathematics. London: Springer. xv, 543 p. (1999).

[GR] B. Grecu, R. Ryan, Polynomials on Banach spaces with unconditional bases, Proc. Amer. Math. Soc. 133 (2005), 1083-1091.

[H] L. Hörmander, An Introduction to Complex Analysis in Several Variables, New York: North-Holland (2000). 
$[\mathrm{J}] \quad$ B. Josefson, Approximation of holomorphic functions in certain Banach spaces, Int. J. Math. 15 (2004), 467-471.

[L1] L. Lempert, Approximations of holomorphic functions of infinitely many variables, II, Ann. Inst. Fourier 50 (2000), 423442 .

[L2] L. Lempert, Plurisubharmonic domination, J. Am. Math. Soc. 17 (2004), 361-372.

[L3] L. Lempert, Analytic sheaves in Banach spaces, manuscript (2005).

[LT] J. Lindenstrass, L. Tzafriri, Classical Banach Spaces I and II, New York: Springer (1977).

[M] J. Mujica, Complex analysis in Banach spaces, North-Holland Mathematics Studies, 120. Notas de Matemtica, 107. Amsterdam/New York/Oxford: North-Holland. XI, 434 p. (1986).

[N] R. Narasimhan, Imbeddings of holomorphically complete complex spaces, Am. J. Math. 82 (1960), 917-934.

[P] I. Patyi, Analytic cohomology in a Banach space, manuscript (2005).

[Re] R. Remmert, Habilitationsschrift, Münster (1958).

[Ry] R. Ryan, Introduction to Tensor Products of Banach Spaces, Springer Monographs in Mathematics. London: Springer. xiv, 225 p. (2002). 